\title{
Análisis de la transmitancia térmica y resistencia al impacto de los muros de quincha
}

\section{Analysis of thermal transmittance and resistance to soft shock in wattle walls}

\author{
G. Cuitiño $^{(*)}$, A. Esteves ${ }^{(*)}$, G. Maldonado ${ }^{(* *)}$, R. Rotondaro ${ }^{(* * *)}$
}

\section{RESUMEN}

La quincha es una técnica mixta de muros perteneciente a la familia de los entramados. Compuesta de una armazón estructural (madera o bambú) rellena con barro en estado plástico con añadido de fibras vegetales. Se determinó que la transmitancia térmica de un panel de quincha de $0,10 \mathrm{~m}$ de espesor, dando como resultado 2,64 W/m² $\mathrm{K}$ y con el agregado de una placa de poliestireno expandido de $5 \mathrm{~mm}$ de espesor en el interior el panel, la transmitancia es $2,02 \mathrm{~W} / \mathrm{m}^{2} \mathrm{~K}$. Estos valores son comparables al de un muro de ladrillo de $0,20 \mathrm{~m}$ de espesor y revocado en ambas caras, cuyo valor es 4,13 W/ $\mathrm{m}^{2} \mathrm{~K}$. Para la resistencia al impacto, se construyeron paneles en escala 1:1 y se ensayaron al choque blando. Las deformaciones permanentes fue en todos los casos menor de 3,6 mm requerido por la Norma vigente. Esto implica que no sufrió deformaciones apreciables dando seguridad a los cerramientos de una vivienda. Se concluye que su buen comportamiento térmico y estructural, sumado a las características de ser económico y apropiado para la autoconstrucción, lo hacen viable para implementar a escala masiva.

Palabras claves: Quincha; transmitancia térmica; impacto en muros.

\section{ABSTRACT}

Wattle construction is a mixed technique used on walls and that belong to the frameworks family. It is constructed with a structural frame (wood or bamboo) filled in with a mixture of clay mud in a plastic state and vegetable fiber. In this paper thermal transmittance and soft shock resistance had been studied, thermal transmittance had been measured with wattle samples of $0.10 \mathrm{~m}$ thickness, and result in $2.64 \mathrm{~W} / \mathrm{m}^{2} \mathrm{~K}$ and with the addition of expanded polystyrene sheet of 5 mm thicknees inside the panel, the transmittance was $2.02 \mathrm{~W} / \mathrm{m}^{2} \mathrm{~K}$. These values are batter than a brick wall of o.2O $\mathrm{m}$ thick with a finish plaster on both sides of the wall, whose value is $4.13 \mathrm{~W} / \mathrm{m}^{2} \mathrm{~K}$. For impact resistance, panels were constructed and tested to soft shock, on 1:1 scale. The permanent deformation in all cases was less than 3.6 mm required by the current standard. This implies that there was no appreciable deformations suffered enclosures providing security to a home. It's concludes that has a good thermal and structural behavior, plus the characteristics of being economical and appropriate for selfconstruction, make feasible to implement as a massive scale.

Keywords: Wattle; thermal transmittance; impact on walls.

(*) Centro Científico Tecnológico (CCT) Mendoza - CONICET. Mendoza (Argentina).

(**) Centro Regional de Desarrollos Tecnológicos para la Construcción, Sismología e Ingeniería Sísmica. Mendoza (Argentina).

(***) FADU-Universidad de Buenos Aires. Buenos Aires (Argentina).

Persona de contacto/Corresponding author: gcuitino@mendoza-conicet.gov.ar (G. Cuitiño)

Cómo citar este artículo/Citation: Cuitiño, G., Esteves, A., Maldonado, G., Rotondaro, R. (2015). Análisis de la transmitancia térmica y resistencia al impacto de los muros de quincha. Informes de la Construcción, 67(537): eo63, doi: http://dx.doi.org/10.3989/ic.12.082.

Licencia / License: Salvo indicación contraria, todos los contenidos de la edición electrónica de Informes de la Construcción se distribuyen bajo una licencia de uso y distribución Creative Commons Reconocimiento no Comercial 3.o. España (cc-by-nc). 


\section{INTRODUCCIÓN}

Desde los comienzos de la historia y aún hasta hoy, el hombre utilizó la tierra para construir su cobijo, demostrando una gran capacidad en el manejo de la tierra cruda como material de construcción. Permitiéndoles construir desde humildes viviendas rurales hasta grandes monumentos que datan de miles de años (1).

Diferentes culturas del mundo, con el uso de las técnicas tales como el tapial, el cob, la quincha, bloques de tierra comprimida, han podido crear obras como la ciudad de Jericó (Israel), la aldea Katal Hüyuk (Turquía), las ciudadelas de la cultura Chimú en Chan Chan (Perú) y la tapia reforzada de Joya de Cerén (El Salvador). Esta tecnología, representa en muchas sociedades, una expresión cultural que los relaciona con la naturaleza, la historia cultural y satisface las necesidades que tienen de poseer su propia vivienda.

El uso de la tierra, se ha visto relegado a causa de su asociación con estados de pobreza e insalubridad. Esto ha llevado a que fuera reemplazado por materiales cada vez más manufacturados, es tan así que en las ciudades al momento de construir, la primera opción es el hormigón y la mampostería de ladrillos cocidos, sobretodo en lugares sísmicos. Actualmente, la demanda de viviendas crece rápidamente, y no en todos los casos se puede tener acceso a una vivienda tradicional, de hormigón y ladrillo. Esto genera un aumento del déficit habitacional que se incrementa año a año.

Existen diferentes técnicas de construcción con tierra, una de ellas es la que se conoce como «Entramados» o «Técnicas mixtas», debido a que emplea la madera principalmente como estructura de soporte, la misma luego es recubierta con la tierra a modo de revoque. Este «entramado» adquiere nombres variados en los distintos países latinoamericanos. Así por ejemplo en los de zona andina (Ecuador, Perú, Bolivia) llegando hasta el norte de la Argentina se lo llama quincha, en el Brasil se lo denomina taipa y en otros países va cambiando de nombre (palo a pique, estaqueo, pared francesa, estanteo, entre otros), aunque en muchos de ellos es mejor conocido con el nombre de bahareque (2).

Las construcciones de quincha se presentan como una alternativa a estos problemas. Quincha en el vocabulario Quechua, está vinculada con el uso de la caña para la construcción (3). Este sistema constructivo se caracteriza, mayormente, por la conformación de los cerramientos. En este caso se trabaja con la quincha prefabricada que consiste básicamente en el «empleo de bastidores de madera aserrada, rellenados con cañizo redondo, caña brava o tiras de bambú, todos ellos co- locados en el bastidor en forma trenzada para su autofijación sin necesidad de usar clavos; estos paneles, después de ser montados y fijados en sitio constituyendo paredes, son revocados con barro mezclado con paja, formando una primera capa; $y$, finalmente, reciben una última capa de revoque utilizándose materiales como el barro, cemento, yeso u otro» (4). Para este análisis se empleó un entramado de cañas de Castilla (Arundo donax), el cual luego va recubierto con una mezcla de barro a base de arcilla, arena y fibra vegetal, de acuerdo a lo indicado por Cuitiño (5), lo cual convierte a la quincha en una Construcción liviana y flexibilidad que mejora su capacidad de respuesta al sismo si la comparamos con otras técnicas convencionales de mampostería estructural (6).

Las viviendas de quincha, además de su alta flexibilidad, presentan ventaja en su comportamiento térmico, permitiéndole a los muros resguardar los ambientes interiores del calor durante el día y en el transcurso de la noche de las bajas temperaturas exteriores (7). Sin embargo, no se cuenta con valores de transmitancia térmica medidos en muros reales que permita un conocimiento más preciso al momento de realizar un balance térmico.

La transmitancia térmica (U), es el flujo de calor, en régimen estacionario, dividido por el área y por la diferencia de temperaturas de los medios situados a cada lado del elemento constructivo considerado, siendo su reciproca la resistencia térmica (R).

En el caso de los materiales como el adobe, o la tapia, cuya conformación es más homogénea, la transmitancia térmica se puede calcular a partir de los datos existentes en tablas y del espesor del muro. Sin embargo, en el caso de las construcciones con quincha por tener una conformación más heterogénea, no es posible definir adecuadamente su conductividad térmica a partir de las propiedades de los materiales que la conforman.

Comúnmente el aspecto térmico se ve relegado al momento de construir, debido a que cobran más relevancia temas tales como el costo económico o la situación socio-cultural. Sin embargo, la respuesta térmica de una vivienda tiene influencia sobre las personas que viven en ella. Es importante que la habitabilidad del interior de la vivienda sea la adecuada para lograr el confort térmico y de esa forma, proporcionar una mejora en la calidad de vida.

En la Tabla 1 se muestran algunos de los valores de conductividad térmica de los materiales más empleados en la construcción, analizados por diversos autores. Se observa que la conductividad térmica de los muros de tierra comprimida o tapial,

Tabla 1. Conductividad térmica de los materiales.

\begin{tabular}{|l|r|c|c|}
\hline Material & & Densidad [kg/m & Conductividad [W/mK] \\
\hline Tapial & fuente (8) & 1.400 & 0,60 \\
\hline Btc & $(8)$ & 1.700 & 0,81 \\
\hline \multirow{3}{*}{ Adobe } & $(8)$ & 1.200 & 0,46 \\
\cline { 3 - 4 } & $(9)$ & 1.650 & 0,82 \\
\cline { 3 - 4 } & $(6)$ & 750 & 0,20 \\
\hline Balas de paja & $(10)$ & 60 & 0,067 \\
\hline Quincha prefabricada & $(11)$ & 1289 & 0,17 \\
\hline Hormigón normal & $(12)$ & 2.400 & 1,63 \\
\hline Pared de ladrillo macizo & $(12)$ & 1.800 & 0,91 \\
\hline
\end{tabular}


el bloque de tierra comprimida (Btc) y adobe son menores que la del hormigón y el ladrillo, es decir que son mejores aislantes, siendo que la resistencia térmica de un muro de adobe de 300 $\mathrm{mm}$ de espesor es de $0,65 \mathrm{~m}^{2} \mathrm{~K} / \mathrm{W}$, mientras que para el mismo muro de hormigón armado la resistencia sería $0,18 \mathrm{~m}^{2} \mathrm{~K} / \mathrm{W}$, lo cual es considerablemente menor, y si se pretendiera tener una resistencia similar a la del muro de adobe, seria necesario trabajar con un espesor de muro de 1,06 m.

Existen trabajos que indican la conductividad térmica de la caña (13), empleada en los cerramientos de los muros, dispuestas una al lado de la otra, formando una especie de encofrado. A partir de este dato, se podría contar con la resistencia térmica de las cañas, pero serviría únicamente cuando las mismas están juntas unas con otras, como es el caso de la quincha prefabricada expresada en la Tabla 1, sin embargo la conformación de los muros de quincha en estudio es diferente, debido a que las cañas se disponen formando un enrejado dejando espacios de $30 \mathrm{~mm}$ entre ellas. Por lo tanto, al existir escasa bibliografía que considere los aspectos constructivos específicos de esta tecnología, resulta necesario realizar mediciones experimentales de la transmitancia térmica en muestras construidas de igual manera que los paneles en estudio.

Desde el punto de vista estructural, de acuerdo a la Norma IRAM 11.595, uno de los ensayos que se debe realizar en muros opacos de cerramiento, es el de choque blando, descrito en la Norma IRAM 11.596. Este ensayo permite conocer la energía de impacto necesaria para dañar un muro de quincha, lo que puede relacionarse con la resistencia al viento y la seguridad habitacional (14). No existen datos de resistencia al choque para los muros construidos con quincha.

\section{OBJETIVOS}

En el presente trabajo se presenta la evaluación térmica y estructural, realizada en muros de quincha prefabricada. El mismo, se encuentra en el marco de la tesis doctoral titulada: Arquitectura en zonas sísmicas. Estudio energético, ambiental y técnico económico de construcciones sustentables con quincha. Durante el desarrollo de esta tesis se construyeron paneles a escala 1:1 con la tecnología de quincha para sus posteriores ensayos en Instituciones regidas por normas. Se pretende a través del mismo evaluar y mejorar la tecnología existente desde los puntos de vista térmico, estructural y de acabado superficial. Los objetivos específicos son los siguientes:

a- Determinar la mejor composición de suelo para ser utilizada como relleno de los muros de quincha para viviendas en la zona del Centro-Oeste de Argentina.

b- Determinar la transmitancia térmica de los cerramientos de quincha prefabricada sin y con mejoras térmicas. c- Determinar la resistencia al impacto de choque blando de los paneles de quincha, para su posterior empleo en los cerramientos verticales en viviendas.

\section{METODOLOGÍA}

Proceso de selección de suelos: Para el desarrollo del análisis de los muros de quincha, se determinó la mejor composición de suelo para realizar el relleno de los muros. Se seleccionaron cuatro zonas de la provincia de Mendoza - Argentina, donde los suelos presentaban un cierto contenido de arcilla, a saber: Malargüe, Villa 25 de Mayo, Salto de las Rosas y Lavalle (15). A partir de la extracción, se los clasificó por el Sistema Unificado de Clasificación de Suelos, y se obtuvo para todos los casos una composición franco arcillo - arenoso. Con cada uno de los suelos, se armaron tres probetas de $4 \mathrm{~cm} \times 4 \mathrm{~cm}$ $\times 16 \mathrm{~cm}$ de largo, de esta forma se obtiene un valor medio de la resistencia a compresión y de la retracción lineal de las mismas, Figura 1.

Proceso de armado de las probetas: consiste en realizar agregados al suelo en su estado natural de extracción, de arena y de fibra de paja de trigo, hasta lograr resultados satisfactorios (16). Siendo la retracción lineal el ensayo predominante, debido a que se relaciona directamente con la formación de las fisuras durante el secado, si se logra controlar la retracción se podrá evitar la formación de fisuras durante el secado de los muros de quincha y por ende el anidamiento de insectos. A demás se midió también la tensión de compresión para comparar la resistencia de los distintos suelos conformados.

Debido a la heterogeneidad en cuanto a la conformación del material de quincha (presencia de cañas a distinta distancia, listones de madera), se determinó en forma experimental transmitancia térmica de los muros de quincha.

Se empleó la de Castilla (Arundo donax), la misma pertenece a la familia botánica de las Gramíneas (Gramineae). Presenta un tallo que consta de nudos separados por entrenudos ahuecados; crece en grupos compactos y en forma rápida durante el verano. El ciclo de la caña, desde el momento de la brotación hasta su cosecha se cumple en 540 a 640 días aproximadamente. La cosecha se realiza durante el invierno porque es el momento en el cual la savia de la planta está en bajas cantidades en el interior de los tallos (17).

Proceso de montaje de paneles a ensayar: para el ensayo se construyeron cuatro paneles de 0,56 m × 0,56 m $\times 0,094 \mathrm{~m}$ de espesor. El tamaño de los mismos fueron los adecuados de acuerdo al tamaño de la máquina de ensayo. Se realizó una terminación superficial plana, de esta forma la superficie de contacto con las placas de la máquina de ensayo fue total. La evaluación térmica se realizó en el Laboratorio del Centro de

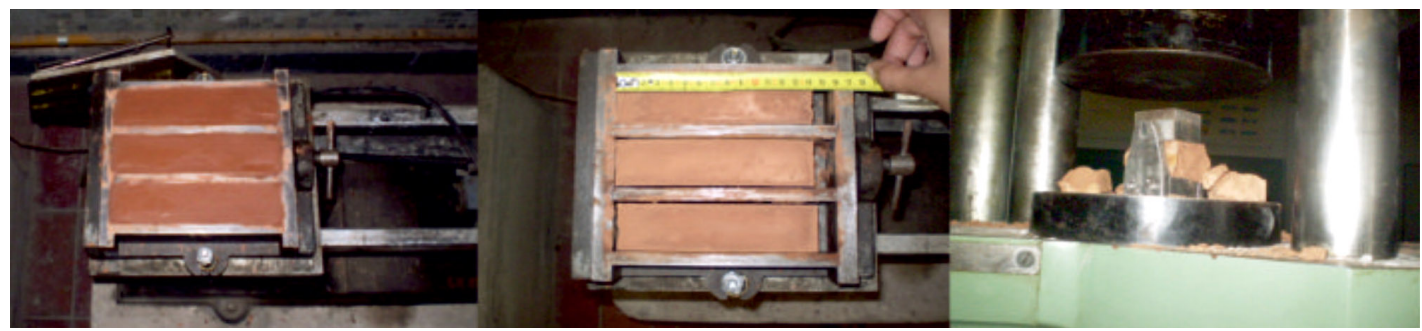

Figura 1. Secuencia de ensayo en probetas: Armado de probetas de $4 \mathrm{~cm} \times 4 \mathrm{~cm} \times 16 \mathrm{~cm}$, medición de retracción lineal y ensayo de compresión. 
Investigación y Desarrollo en Construcciones perteneciente al Instituto Nacional de Tecnología Industrial (INTI), ubicado en Buenos Aires - Argentina.

La construcción de las muestras se realizó en el Laboratorio de Ambiente Humano y Vivienda, perteneciente al Instituto de Ciencias Humanas, Sociales y Ambientales del Centro Científico y Tecnológico CONICET - Mendoza. Por cada ensayo se requieren dos paneles para poder obtener un valor promedio de transmitancia térmica de superficie a superficie, en total se construyeron cuatro paneles.

La construcción de los paneles 1 y 2 consistió en un bastidor de álamo de 1 " × 4", al mismo se le clavó en el interior un listón de álamo de 1 1/2" × 1/2", en forma equidistante de los bordes. A este listón se le clavan las cañas de ambos lados del listón interno. De este modo, queda un entramado con 16 cañas en el sentido longitudinal en una cara y 15 cañas en el sentido transversal en la otra cara (ver Figura 2). Los diámetros de las cañas oscilan entre los $16 \mathrm{~mm}$ y $25 \mathrm{~mm}$, siendo la separación entre ejes de las cañas de 0,035 m. De esta forma se obtuvo un entramado que permite introducir el barro con facilidad. El relleno en ambas caras es una mezcla a base de tierra arcillosa, arena y paja de trigo. La tierra arcillosa proviene de Salto de las Rosas. La paja de trigo que se incorpora tiene $10 \mathrm{~cm}$ de longitud. El revoque final que tiene $2 \mathrm{~mm}$ de espesor, es de arena, arcilla y cal (para otorgarle la terminación plana que se requiere para el ensayo). Cabe destacar que se ha tratado de reproducir lo más fielmente posible la forma de construir el panel que luego se pretende materializar en una vivienda.

Los paneles 3 y 4 poseen iguales dimensiones y relleno de mezcla de tierra, arena y paja, que los paneles 1 y 2 , excepto que en este caso, se dispuso de una capa rígida de poliestireno
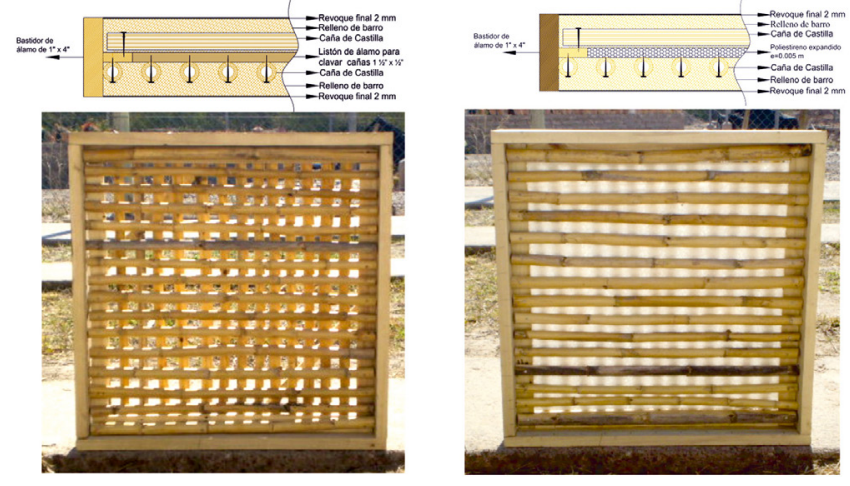

Figura 2. Armado de los paneles de quincha sin y con aislamiento térmico para su posterior llenado con barro. Ancho del panel o,6 m × o,6 m, diámetro de las cañas de Castilla 0,025 m, clavos negros punta paris. expandido de 0,005 $\mathrm{m}$ de espesor, como aislamiento térmico adicional, en el centro del panel entre ambas caras del entramado y el revoque final es a base de cemento, Figura 3 .

Proceso de ensayo de paneles: para la evaluación térmica se determinan los valores de Transmitancia Térmica (K) a $25{ }^{\circ} \mathrm{C}$ de temperatura media, de acuerdo a las Normas:

- Norma ISO 8302: Thermal insulation. Determination of steady state thermal resistance and related properties. Guarded hot plate apparatus (18).

- ASTM C177: Standard Test Method for Steady - State Heat Flux Measurements and Thermal Transmission Properties by means of the Guarded Hot Plate Apparatus (19).

- IRAM 11559: Determinación de la conductividad térmica y propiedades conexas en régimen estacionario. Método de la placa caliente con guarda (20).

Con respecto a la resistencia estructural de la construcción con quincha, el ensayo de choque blando, de acuerdo a la norma ASTM 695 (21), proporciona datos que pueden ser utilizados para evaluar el rendimiento relativo de pared, suelo, techo y construcciones en condiciones representativas de las sufridas en el servicio real cuando se somete a impacto con un objeto contundente pesado.

Para el ensayo del panel se sujetó tanto en la parte inferior como la superior, dejando la parte central del mismo libre para realizar el ensayo. De acuerdo a la Norma IRAM 11596 (22), se empleó un saco de cuero conteniendo en su interior arena, totalizando peso conjunto (saco + arena) de $30 \mathrm{~kg}$ (23). El mismo debe impactar en la zona que presente menor resistencia, por lo tanto se realiza el impacto en uno de los paños centrales de la quincha y se mide la deformación en el centro geométrico del panel, el cual coincide con la diagonal conformada por un listón de madera, Figura 4.

Proceso de armado de los paneles para choque blando: se construyeron cuatro paneles de 1,20 $\mathrm{m} \times 1,80 \mathrm{~m} \times 0,10 \mathrm{~m}$, (Figura 5), debido a que la máquina de ensayo responde a estas medidas. Por otro lado, también responden a una habitación modulada de $2,80 \mathrm{~m} \times 2,80 \mathrm{~m}$ ó $2,80 \mathrm{~m} \times 4,20 \mathrm{~m}$, con la ayuda de columnas de rollizos de aproximadamente o,20 m de diámetro, donde a la altura de 1,80 m del panel se suma el zócalo de ladrillo o piedra que debe ir por debajo para alcanzar los 2,40 $\mathrm{m}$.

El bastidor del panel se compone de listones en toda la periferia mas una diagonal y dos listones horizontales de madera de álamo, dando como resultado un panel con seis paños ciegos, Figura 5. Se han tomado los dos centrales para el desarrollo del ensayo, debido que son los que presentan características más desfavorables dada su menor resistencia
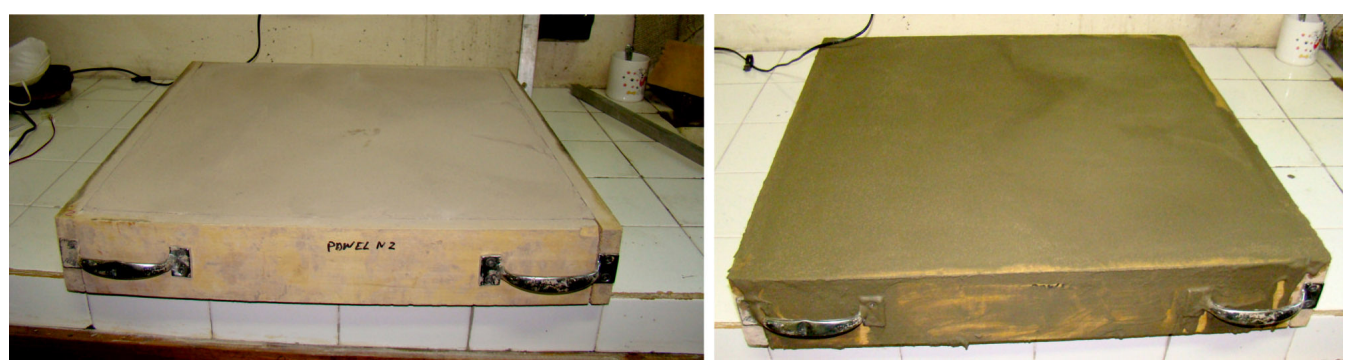

Figura 3. Paneles de quincha rellenos con barro y revocados para conformar una superficie lisa para el ensayo. 

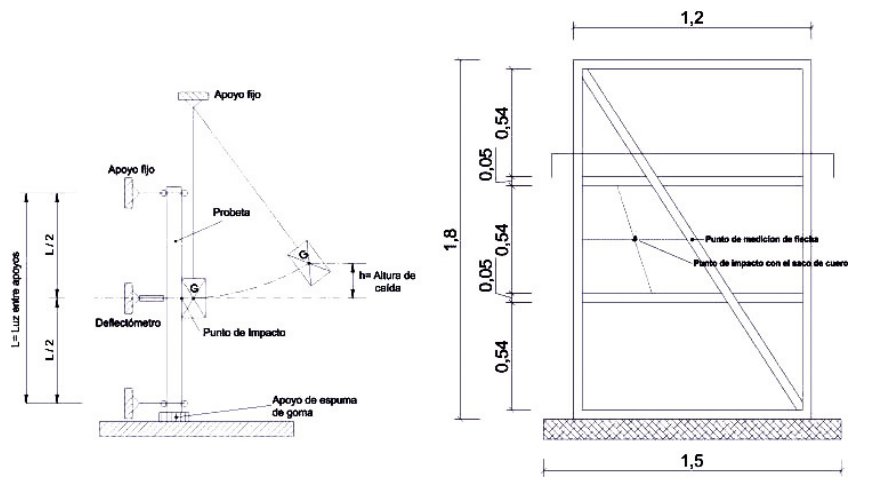

Figura 4. Diseño del panel de ensayo de choque blando.

respecto de centro geométrico del panel, el cual se encuentra sobre la diagonal del mismo (24).

Los paneles se construyeron con listones de álamo cepillado de 2 " $\times 4$ ". En el interior se dispuso de un listón de 1 1/2" $\times$ $1 / 2$ " al cual se sujetaron las cañas de Castilla, al igual que en el caso anterior. El diámetro exterior de las mismas oscila entre los $20 \mathrm{~mm}$ y $30 \mathrm{~mm}$, las mismas se disponen en sentido vertical en una cara del panel y en sentido horizontal en la cara opuesta.

Como recubrimiento se empleó una mezcla de barro de iguales características que las empleadas en el panel para el ensayo de transmisión térmica. Debido a los requerimientos del ensayo de choque blando se dispuso de un rollizo de álamo de o,10 $\mathrm{m}$ de diámetro en la base del panel para que sirviera de apoyo. Finalmente se pintó todo el panel con pintura de esmalte sintético blanco, para poder observar respuestas, tales como microfisuras o desprendimientos del panel durante el ensayo correspondiente.

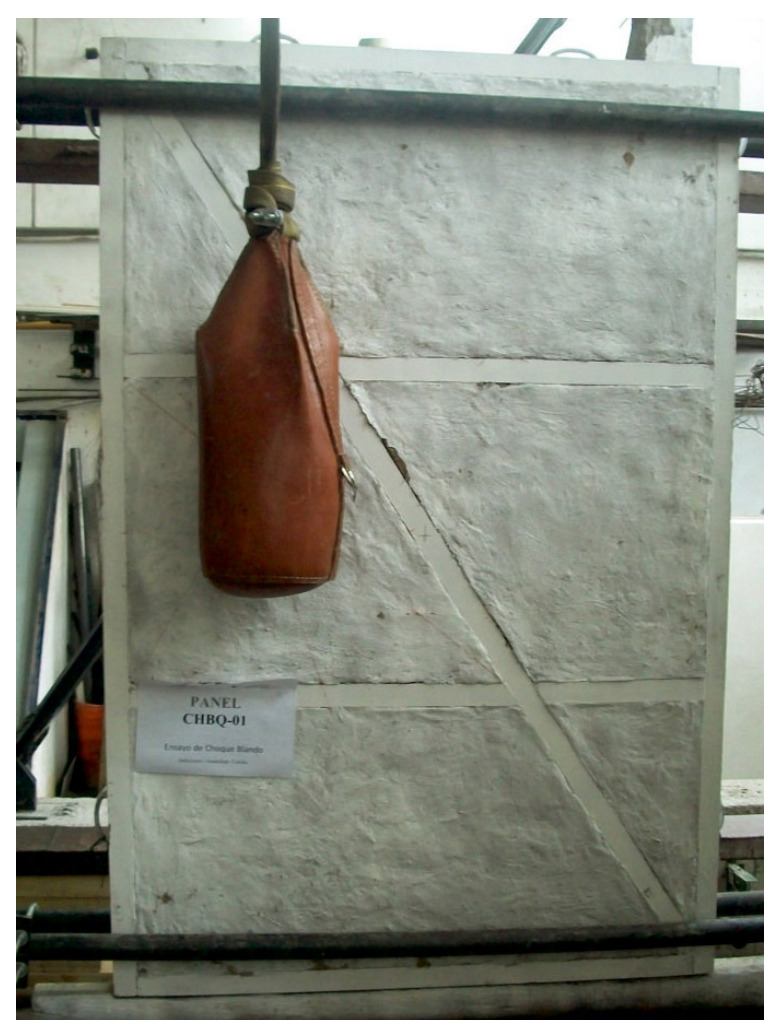

Figura 5. Panel de quincha para ensayo de choque blando, saco de cuero con $30 \mathrm{Kg}$ de arena empleado en el ensayo.
Proceso de ensayo de los paneles: para la determinación de la Resistencia al choque blando, se emplearon las Normas:

- IRAM 11.596/o7: Método de ensayo de impacto sobre probeta vertical. (22)

- IRAM 11.585/91: Paneles para muros y tabiques en edificios. (25)

\section{CRITERIO DE SELECCIÓN DE SUELOS}

La extracción de los suelos, se realizó en cuatro zonas de Mendoza, donde se tenía conocimientos por los habitantes del lugar, que los mismos presentaban características de suelos arcillosos, siendo además que coincidían con las zonas potenciales de suelos finos. Asimismo durante la toma de muestras se realizó una verificación sensorial de los suelos, que consistió en: caracterización por tamaño de las partículas, por color, por brillo y por tacto. Los resultados de estos ensayos dieron para todos los casos de suelos extraídos, que había presencia de suelos finos, lo que justificó estudiar las muestras suelo, trasladando aproximadamente $30 \mathrm{~kg}$ al laboratorio y realizar su clasificación.

Una vez que se definen las nuevas proporciones de cada suelo, con el agregado de arena y la fibra vegetal, se agrega agua hasta obtener una mezcla plástica y se procede a armar las probetas, para los ensayos de retracción lineal y de tensión de compresión, se dejan secar a la temperatura ambiente, durante aproximadamente 28 días o hasta que se logre peso constante. En la Tabla 2, se puede apreciar las proporciones de las mezclas de suelo que se obtuvieron para cada caso de estudio. En el caso del suelo de Lavalle, no se realizó el último aumento de arena debido a que la mezcla perdía demasiada cohesión.

Tabla 2. Proporciones de mezclas de suelos.

\begin{tabular}{|c|c|c|c|}
\hline & 1 & 2 & 3 \\
\hline \multicolumn{4}{|c|}{ Malargüe } \\
\hline \% Suelo original & 87,50 & 75,00 & 50,00 \\
\hline \% Arena Agregada & 12,50 & 25,00 & 50,00 \\
\hline \% Fibra Vegetal & 0,00 & 1,00 & 1,50 \\
\hline \multicolumn{4}{|c|}{ Salto de las Rosas } \\
\hline \% Suelo original & 85,71 & 71,43 & 42,86 \\
\hline$\%$ Arena Agregada & 14,30 & 28,57 & 57,14 \\
\hline \% Fibra Vegetal & 0,00 & 1,00 & 1,50 \\
\hline \multicolumn{4}{|c|}{ Lavalle } \\
\hline \% Suelo original & 66,66 & 33,34 & \\
\hline$\%$ Arena Agregada & 33,34 & 66,66 & \\
\hline \% Fibra Vegetal & 0,00 & 1,00 & \\
\hline \multicolumn{4}{|c|}{ Villa 25 de Mayo } \\
\hline \% Suelo original & 83,30 & 66,66 & 33,34 \\
\hline \% Arena Agregada & 16,70 & 33,34 & 66,66 \\
\hline \% Fibra Vegetal & 0,00 & 1,00 & 1,50 \\
\hline
\end{tabular}

1- Aumento del $5 \%$ de arena.

2- Aumento del $10 \%$ de arena más el $1 \%$ de fibra vegetal.

3- Aumento del $20 \%$ de arena más el 1,5\% de fibra vegetal.

La retracción lineal se encuentra relacionada con la fisuración que podría sufrir el revoque de tierra una vez colocado en el muro de quincha. Se observa como se reduce la retracción al agregar el $1 \%$ en peso de paja de trigo en todos los casos, Figura 6. La resistencia de compresión disminuyen con el agregado de arena y de fibra, sin embargo el agregado de arena y fibra resulta necesaria para otorgarle a la mezcla 


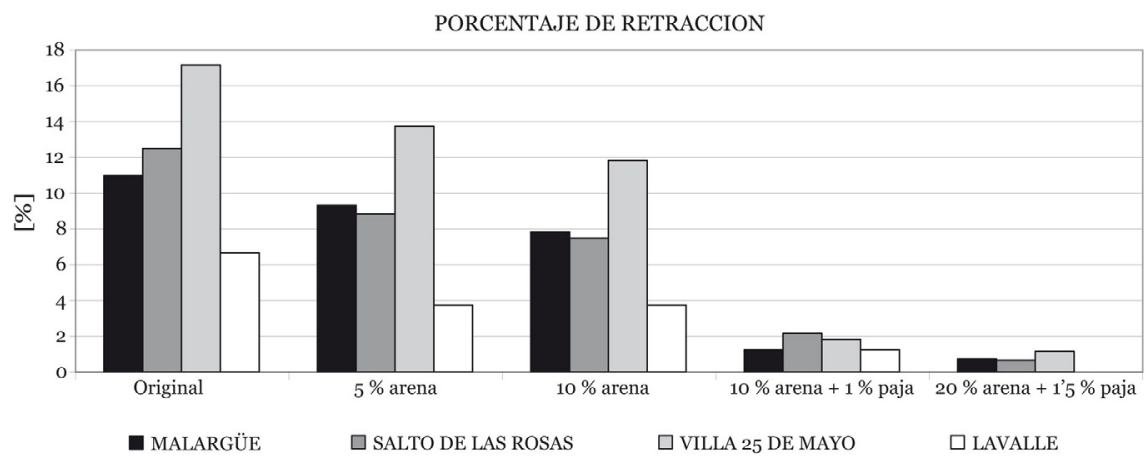

Figura 6. Porcentaje de retracción lineal de los suelos ensayados.

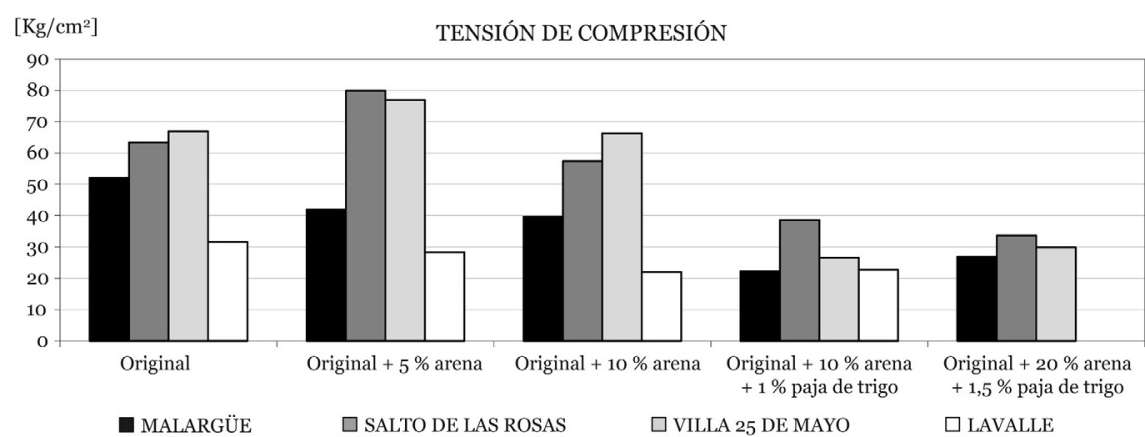

Figura 7. Tensión de compresión de los suelos ensayados.

más estructura y ayudar a reducir la fisuración. Estos datos constituyen una guía inicial sobre cómo resistirá el barro ante solicitaciones compresión una vez puesto en la pared de quincha, sin el uso de estabilizantes químicos, Figura 7.

La mezcla que mejor resultó fue la correspondiente al suelo extraído de Salto de las Rosas más un agregado del $20 \%$ del peso del suelo original en arena y el 1,5\% del peso del suelo original en peso de fibra vegetal. Se observa que tiene la mejor combinación entre la menor retracción lineal y mayor tensión de compresión, respecto de los otros suelos ensayados bajo las mismas proporciones.

\section{ENSAYO DE TRANSMITANCIA TÉRMICA}

Una vez elegida la mezcla de suelo con mejor comportamiento, se procedió al armado de los paneles de quincha para determinar la transmitancia térmica. Con los paneles ya terminados, se determinó cambio relativo de la masa de los paneles, para ello se los dejó secar en un horno durante 936 horas a $60^{\circ} \mathrm{C}$ hasta llegar a peso constante, luego se los dejó aclimatar bajo las condiciones ambientales del laboratorio, hasta alcanzar el equilibrio higrotérmico con el mismo. En el caso del panel 3, durante el secado en el horno se desprendió el revoque exterior de cemento, por ello se procedió a revocar nuevamente pero en este caso la superficie de barro se dejó sin mucho alisado para permitir una mejor unión entre el revoque de cemento y el barro, y para que ambos paneles tuvieran las mismas condiciones también se rehizo revoque del panel 4. Se los dejó secar a temperatura ambiente (Temperatura ambiente: $24^{\circ} \mathrm{C}$; Humedad relativa: $53 \%$ ) por $24 \mathrm{~h}$ y luego se procedió a realizar el acondicionamiento higrotérmico, que en este caso fue durante 624 horas. Los datos obtenidos de esta etapa de secado, se encuentran indicados en la Tabla 3.

Donde los datos representados en la Tabla 3 son:

$\mathbf{M}_{1}$ es el peso de los paneles en su estado inicial.

$\mathbf{M}_{2}$ es el peso de los paneles luego de sufrir un proceso de secado en hornos a una temperatura de $60^{\circ} \mathrm{C}$, hasta que tenga peso constante.

$\mathbf{M}_{3}$ es el peso con el que quedan los paneles luego de dejarlos bajo las condiciones ambientales del laboratorio hasta que hasta que han alcanzado la humedad de equilibrio.

A partir de estos datos se pudo determinar el cambio de masa que sufrieron los paneles luego del proceso de secado $\left(\mathrm{m}_{\mathrm{r}}\right)$, el debido al proceso de acondicionamiento térmico $\left(\mathrm{m}_{\mathrm{c}}\right)$, finalmente se determina el cambio relativo de masa debido únicamente al acondicionamiento higrotérmico luego del proceso de secado $\left(\mathrm{m}_{\mathrm{d}}\right)$, dichos valores se obtienen de las ecuaciones [1], [2] y [3].

Tabla 3. Variación de la masa de los paneles durante el proceso de secado.

\begin{tabular}{|c|c|c|c|c|c|c|c|}
\hline \multirow[b]{2}{*}{ Designación } & \multirow{2}{*}{$\begin{array}{c}\text { Tiempo de secado } \\
{[\text { [h] }}\end{array}$} & \multicolumn{3}{|c|}{ Masa de la probeta } & \multicolumn{3}{|c|}{ Cambio relativo de masa del Panel } \\
\hline & & $\underset{[\mathrm{Kg}]}{\mathbf{M}_{1}}$ & $\underset{[\mathbf{K g}]}{\mathbf{M}_{2}}$ & $\underset{[\mathbf{K g}]}{\mathbf{M}_{3}}$ & $\mathbf{m}_{\mathrm{r}}$ & $\mathbf{m}_{\mathrm{c}}$ & $\mathbf{m}_{\mathrm{d}}$ \\
\hline Panel 1 & 936 & 36,79 & 35,48 & 35,76 & 3,7 & 2,9 & 0,8 \\
\hline Panel 2 & 936 & 38,24 & 35,89 & 36,15 & 6,6 & 5,8 & 0,7 \\
\hline Panel 3 & 624 & 37,07 & 35,39 & 35,42 & 4,8 & 4,7 & 0,08 \\
\hline Panel 4 & 624 & 46,94 & 34,97 & 35,00 & 34,2 & 34,1 & 0,09 \\
\hline
\end{tabular}




$$
\begin{aligned}
& m_{r}=\frac{M_{1}-M_{2}}{M_{2}} \\
& m_{c}=\frac{M_{1}-M_{3}}{M_{3}} \\
& m_{d}=\frac{M_{3}-M_{2}}{M_{2}}
\end{aligned}
$$

La poca variación de la masa en los paneles de quincha durante este proceso indican que la absorción de humedad en el acondicionamiento higrotérmico es reducido, siendo esta una ventaja al momento de evaluar la transmitancia térmica de los muros, ya que a mayor humedad, mayor será la transferencia de calor por conducción del muro.

Para la medición de la transmitancia térmica de los paneles de quincha se hizo uso del sistema de placa caliente normalizado (18), (19) y (20). El mismo consiste en colocar en forma horizontal los dos paneles de quincha fabricados con una placa caliente en el medio, una placa fría arriba de la muestra superior y otra placa fría debajo de la muestra inferior y luego se aisló todo el perímetro del panel para evitar las pérdidas de calor por la periferia, en la Figura 8 se puede apreciar la disposición de los paneles al momento de realizar el ensayo. En la Tabla 4, se puede observar que para el análisis de transmitancia térmica, se trabajó con un rango de temperatura media de placa caliente de $37^{\circ} \mathrm{C}$ y $13{ }^{\circ} \mathrm{C}$ en el caso de la placa fría.

La expresión matemática que permite conocer la transmitancia térmica promedio de los dos paneles de quincha, se plantea como una ecuación de balance de energía [4] y [5]:

$$
Q=q_{1}+q_{2}
$$

Aplicando la Ley de Fourier:

$$
Q=\left[\frac{k_{1} \times A \times \Delta T_{1}}{e_{1}}+\frac{k_{2} \times A \times \Delta T_{2}}{e_{2}}\right] \times \frac{1}{e_{m}}
$$

Donde:

Q: Potencia térmica; $\mathrm{V} \times \mathrm{I} \times \mathrm{N}\left[\mathrm{W} / \mathrm{m}^{2}{ }^{\circ} \mathrm{C}\right]$
V: Tensión suministrada [V].

I: Corriente suministrada [A].

$\mathrm{N}$ : Factor de calibración del equipo de placa caliente: 0,985

$k_{1-2}$ : Es la transmitancia térmica del panel superior e inferior, respectivamente $\left[\mathrm{W} / \mathrm{m}^{2} \mathrm{~K}\right]$.

$A$ : Área de la placa caliente: 0,3048 $\mathrm{m} \times 0,3048 \mathrm{~m}=0,0948$ $\mathrm{m}^{2}$.

$\Delta T_{1-2}$ : Delta de temperatura para el panel superior y para el panel inferior, respectivamente $\left[{ }^{\circ} \mathrm{C}\right]$.

$e_{1-2}$ : Espesor del panel superior e inferior, respectivamente $[\mathrm{m}]$.

De la ecuación [5] se despeja la transmitancia térmica y asumiendo que es la misma para ambos paneles, se obtiene [6]:

$$
k=\frac{Q}{A \times e_{m}} \times\left[\frac{e_{1} \times e_{2}}{\Delta T_{1} \times e_{2}+\Delta T_{2} \times e_{1}}\right]
$$

A partir de la ecuación [6], se obtuvo como resultado de este ensayo, un valor promedio de transmitancia térmica para los dos paneles de $2,64 \mathrm{~W} / \mathrm{m}^{2} \mathrm{~K}$ para el caso de los paneles tradicionales sin agregado de aislamiento, y un valor promedio de $2,02 \mathrm{~W} / \mathrm{m}^{2} \mathrm{~K}$ para el caso de los dos paneles con el agregado de poliestireno expandido, estos valores se encuentran indicados en la Tabla 5 .

\subsection{Análisis de los resultados}

El valor de transmitancia térmica entre ambas superficies, obtenido en forma experimental, es de $2,64 \mathrm{~W} / \mathrm{m}^{2} \mathrm{~K}$ para un muro de quincha, con un espesor de 0,094 $\mathrm{m}$ sin aislamiento térmico. Este dato puede ser comparado con el valor obtenido en forma analítica en el trabajo realizado por Esteban Fernández (26), cuyo cálculo de transmitancia térmica de un panel de quincha de $0,075 \mathrm{~m}$ de espesor, es de $5,17 \mathrm{~W} / \mathrm{m}^{2} \mathrm{~K}$, este valor puede ser mejorado con el consiguiente aumento de espesor del muro. Si se lo relaciona con el valor de transmitancia térmica de un muro de ladrillo revocado en ambas caras de $0,20 \mathrm{~m}$ de espesor y ensayado bajo las mismas condiciones que los paneles de quincha, el valor es de 4,13 W/ $\mathrm{m}^{2} \mathrm{~K}$ (27) o con la de un muro de adobe de o,30 $\mathrm{m}$ de espesor cuyo $\mathrm{K}=2,73 \mathrm{~W} / \mathrm{m}^{2} \mathrm{~K}(9)$, se puede concluir que todos ellos

Tabla 4. Temperaturas de las placas en los ensayos de los paneles.

\begin{tabular}{|l|c|c|}
\cline { 2 - 3 } \multicolumn{1}{c|}{} & Panel 1 - 2 & Panel 3-4 \\
\hline Temperatura de Placa Caliente & $37,0^{\circ} \mathrm{C} \pm 0,1^{\circ} \mathrm{C}$ & $37,2^{\circ} \mathrm{C} \pm 0,1^{\circ} \mathrm{C}$ \\
\hline Temperatura de Placa Fría & $13,2^{\circ} \mathrm{C} \pm 0,1^{\circ} \mathrm{C}$ & $13,2^{\circ} \mathrm{C} \pm 0,1^{\circ} \mathrm{C}$ \\
\hline Espesor promedio de medición & $0,094 \mathrm{~m} \pm 0,0001 \mathrm{~m}$ & $0,094 \mathrm{~m} \pm 0,0001 \mathrm{~m}$ \\
\hline Tensión suministrada & \multicolumn{2}{|c|}{$11,74 \mathrm{~V} \pm 1 \%$} \\
\hline Corriente suministrada & \multicolumn{2}{|c|}{$1,05 \mathrm{~A} \pm 1 \%$} \\
\hline
\end{tabular}
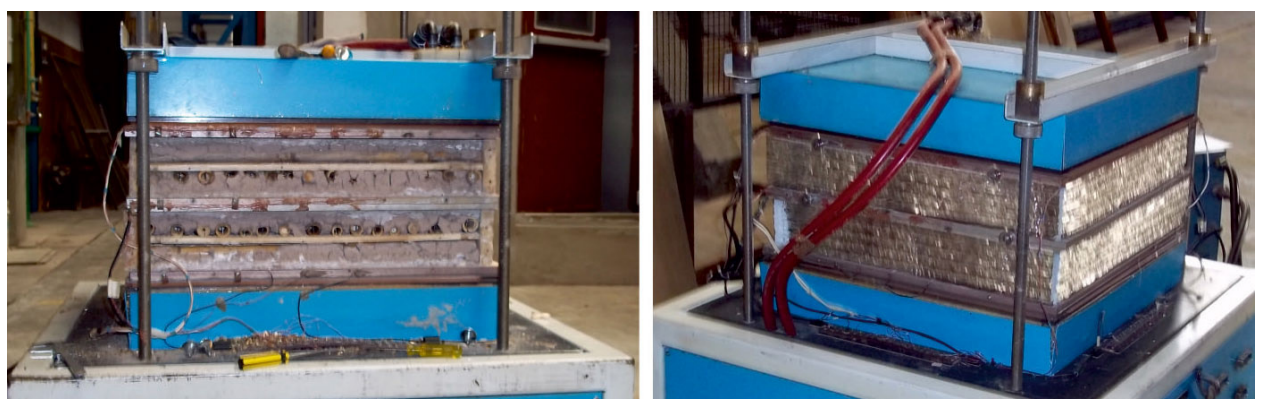

Figura 8. Disposición de los paneles en la máquina de placa caliente en el INTI para el ensayo de transmitancia térmica. 
Tabla 5. Respuesta térmica de los paneles de quincha.

\begin{tabular}{|l|c|c|c|c|c|c|}
\hline \multirow{2}{*}{ Designación } & \multicolumn{2}{|c|}{ Condiciones Ambientales } & \multicolumn{2}{c|}{ Respuesta térmica } & Espesor & Observaciones \\
\cline { 2 - 5 } & $\begin{array}{c}\text { Temperatura } \\
\text { ambiente }\end{array}$ & $\begin{array}{c}\text { Humedad } \\
\text { relativa }\end{array}$ & $\begin{array}{c}\text { Transmitancia } \\
\text { Térmica }\end{array}$ & $\begin{array}{c}\text { Resistencia } \\
\text { Térmica }\end{array}$ & \multirow{2}{\text{[m]}}{} & \\
\hline Panel $1-2$ & $24^{\circ} \mathrm{C}$ & $53 \%$ & $2,64 \mathrm{~W} / \mathrm{m}^{2} \mathrm{~K}$ & $0,38 \mathrm{~m}^{2} \mathrm{~K} / \mathrm{W}$ & \multirow{2}{*}{0,094} & Sin aislamiento \\
\hline Panel $3-4$ & $23,5^{\circ} \mathrm{C}$ & $63 \%$ & $2,02 \mathrm{~W} / \mathrm{m}^{2} \mathrm{~K}$ & $0,49 \mathrm{~m}^{2} \mathrm{~K} / \mathrm{W}$ & Con aislamiento \\
\hline
\end{tabular}

poseen una respuesta térmica similar, siendo además en el caso de los muros de quincha que se emplean materiales reciclables que se encuentran en la naturaleza, al igual que en el caso del adobe. Si embargo este ultimo no tiene las ventajas de la flexibilidad de la quincha para ser empleada en zonas de riesgo sísmico (6).

Así mismo, se puede observar que los valores de transmitancia térmica mejoran cuando al panel se le agrega la placa de poliestireno expandido. En este caso sólo se agregó un placa de poliestireno expandido de 0,005 $\mathrm{m}$ de espesor, pero el valor de $\mathrm{K}=2,02 \mathrm{~W} / \mathrm{m}^{2} \mathrm{~K}$ se podría mejorar aún mas con un espesor mayor de aislante térmico y utilizar la misma tecnología en climas mas rigurosos.

Si a los valores de los muros de quincha obtenidos en forma experimental, les agregamos los coeficientes de película establecidos en las normativas (12), la transmitancia global para el panel sin aislamiento es $1,82 \mathrm{~W} / \mathrm{m}^{2} \mathrm{~K}$ y para el panel con aislamiento es $1,51 \mathrm{~W} / \mathrm{m}^{2} \mathrm{~K}$. Siendo que la provincia de Mendoza corresponde al área bioclimática IV (28), donde las temperaturas mínimas de diseño se encuentran entre $0^{\circ} \mathrm{Cy}-3{ }^{\circ} \mathrm{C}$ y la transmitancia global mínima requerida para invierno es $1,75 \mathrm{~W} / \mathrm{m}^{2} \mathrm{~K}$, se concluye que sólo el muro con aislamiento cumple dicho requisito, razón por la cual se debe continuar investigando en mejoras térmicas.

\section{ENSAYO DE CHOQUE BLANDO}

En los cuatro paneles se procedió a impactar con el saco de arena en el paño de quincha, el saco se lo deja caer en forma pendular (caída libre) desde alturas crecientes de $45 \mathrm{~cm}, 60$ cm y $120 \mathrm{~cm}$, y con la ayuda de un deflectómetro, ubicado sobre la diagonal correspondiente con el punto medio del panel, se miden las flechas instantáneas y permanentes que sufren los paneles, luego de cada impacto, Figura 3.

\subsection{Resultados del ensayo}

A partir de los datos del ensayo con caída libre a diferentes alturas y la masa del saco de cuero de $30 \mathrm{~kg}$, se puede determinar con la ayuda de la ecuación de energía potencial [7], la energía de impacto del saco de cuero contra el panel ensayado.

$$
E=m \times g \times h
$$

Donde:

$E$ : energía de impacto [J]

$m$ : es la masa $[\mathrm{Kg}]$

$g$ : es la aceleración de la gravedad $9,81 \mathrm{~m} / \mathrm{s}^{2}$

$h$ : es la altura de caída [m]

En la Tabla 6 se presentan los datos obtenidos del ensayo de choque blando. Se indica para cada panel la altura de caída, la energía de impacto, la deformación (flecha) permanente e instantánea, los porcentajes requeridos por la norma y los obtenidos en forma experimental y las observaciones. Se puede apreciar la deformación sufrida por el panel al momento del impacto, los mismos se encuentran bajo la denominación de flecha permanente que es la que permanece en el panel una vez finalizado el ensayo y la flecha instantánea que es la que se produce inmediatamente después de realizar el choque.

Los datos obtenidos en las flechas permanentes de los paneles 3 y 4 (valores negativos), están relacionados al hecho de

Tabla 6. Resultados del ensayo de choque blando en paneles de quincha.

\begin{tabular}{|c|c|c|c|c|c|c|c|}
\hline \multirow{2}{*}{$\begin{array}{l}\text { Identificación } \\
\text { de la muestra }\end{array}$} & \multirow{2}{*}{$\begin{array}{c}\text { Impacto } \\
\text { Altura de } \\
\text { caída }[\mathrm{cm}]\end{array}$} & \multirow[b]{2}{*}{ Energía [J] } & \multicolumn{4}{|c|}{ Flecha [mm] } & \multirow[b]{2}{*}{ Observaciones } \\
\hline & & & \% Norma & \% Ensayo & Instantánea & Permanente & \\
\hline \multirow[b]{3}{*}{ Panel 1} & 45 & 135 & 0,20 & 0,02 & 5,10 & 0,4 & Sin deterioro. \\
\hline & 60 & 180 & & & 9,10 & 0,35 & Microfisura horizontal. \\
\hline & 120 & 360 & & & 10,90 & 0,45 & $\begin{array}{l}\text { Pequeño desprendimiento } \\
\text { de barro en marco y paño } \\
\text { ensayado. }\end{array}$ \\
\hline \multirow[b]{3}{*}{ Panel 2} & 45 & 135 & 0,20 & 0,019 & 5,10 & 0,35 & Sin deterioro. \\
\hline & 60 & 180 & & & 9,10 & 0,4 & Microfisura horizontal. \\
\hline & 120 & 360 & & & 10,90 & 0,55 & $\begin{array}{l}\text { Pequeño desprendimiento } \\
\text { de barro en marco y paño } \\
\text { ensayado. }\end{array}$ \\
\hline \multirow{3}{*}{ Panel 3} & 45 & 135 & 0,20 & 0,014 & 5,20 & $-0,25$ & Sin deterioro. \\
\hline & 60 & 180 & & & 8,80 & $-0,45$ & Sin deterioro. \\
\hline & 120 & 360 & & & 12,40 & $-0,55$ & Sin deterioro. \\
\hline \multirow{3}{*}{ Panel 4} & 45 & 135 & 0,20 & 0,005 & 3,50 & $-0,1$ & $\begin{array}{l}\text { Microfisura en unión barro- } \\
\text { madera en el paño ensayado. }\end{array}$ \\
\hline & 60 & 180 & & & 4,00 & $-0,15$ & $\begin{array}{l}\text { Desplazamiento del paño } \\
\text { ensayado. }\end{array}$ \\
\hline & 120 & 360 & & & 4,30 & $-0,1$ & $\begin{array}{l}\text { Desplazamiento importante } \\
\text { del paño ensayado. }\end{array}$ \\
\hline
\end{tabular}



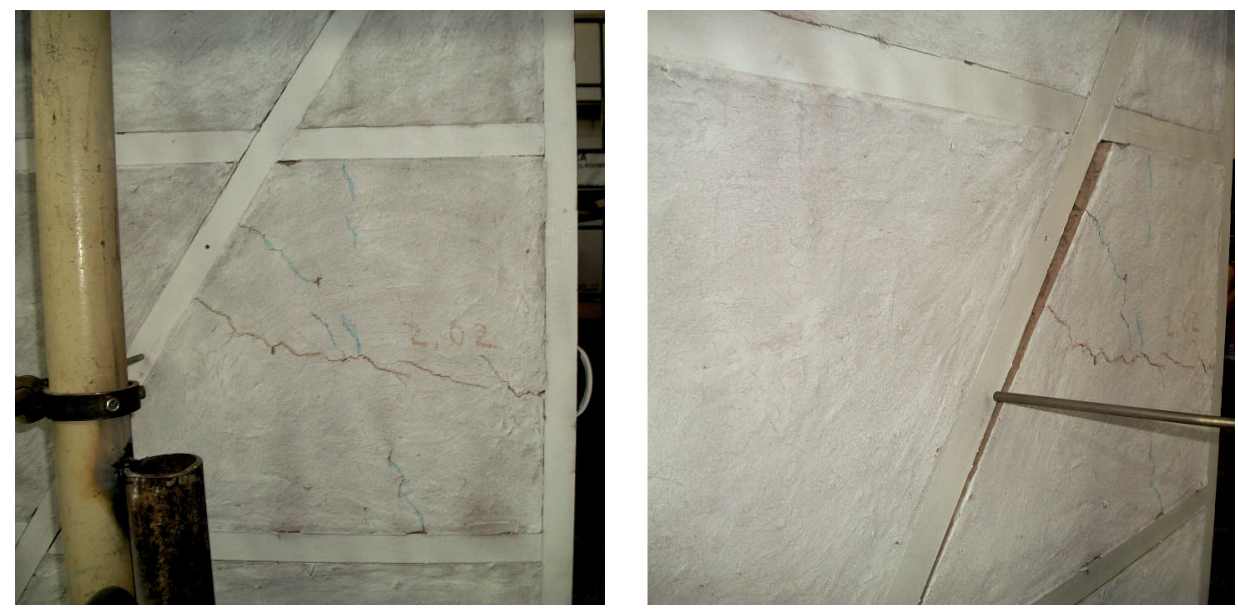

Figura 9. Panel 3 luego del ensayo de choque blando a $360 \mathrm{~J}$, vista frontal y lateral.

que los marcos de los paneles permanecieron con una deformación nula después de realizarse el ensayo de choque. En la Figura 9, se puede apreciar que el paño donde se produce el impacto de choque, la degradación y fisuras formadas en el paño después del choque de $360 \mathrm{~J}$, no compromete la seguridad estructural del panel como para poner en riesgo la seguridad de los ocupantes de la vivienda.

\subsection{Análisis de los resultados}

Según la Norma que respalda el presente ensayo (23), el criterio de aceptación de los paneles bajo la acción del choque blando se debe considerar satisfactorio cuando todas las probetas cumplan con las siguientes condiciones:

a) Deformaciones permanentes: bajo un choque de $135 \mathrm{~J}$, con una altura de caída de la bolsa igual a $45 \mathrm{~cm}$, se debe verificar que la flecha permanente sea menor que el o,2\% de la altura del muro ensayado, que para el caso en estudio es de 3,6 mm y no mayor que $5 \mathrm{~mm}$.

b) Resistencia mecánica: Bajo un choque de $180 \mathrm{~J}$, con una altura de caída de la bolsa igual a o,60 m, el muro no debe sufrir ningún deterioro visible que ponga en riesgo la seguridad de los ocupantes.

c) Bajo el choque de $360 \mathrm{~J}$, con una altura de caída de 120 cm, el muro no debe ser atravesado por la bolsa o ser deteriorado de manera tal que comprometa la seguridad de los ocupantes.
Las deformaciones permanentes sufridas por los paneles ensayados fue en todos los casos menor que el 3,6 mm, requerido por la Norma. Este dato resulta interesante al momento de analizar el comportamiento frente a las deformaciones, siendo importante destacar que a pesar de las microfisuras sufridas en los paños de quincha, donde se realizaron los ensayos de choque, el bastidor de álamo que conforma la pared, no sufrió deformaciones apreciables dando mayor seguridad en lo referente a los cerramientos de una vivienda.

Respecto de la resistencia mecánica, correspondiente a una energía de choque de $180 \mathrm{~J}$ y $360 \mathrm{~J}$, los tres primeros paneles no presentaron daños que pusieran en riesgo la seguridad de los ocupantes, asimismo tampoco fueron atravesados por la bolsa de cuero. Sin embargo el cuarto panel no fue atravesado por el saco pero si resultó deteriorado notablemente, debido a que durante el choque de $180 \mathrm{~J}$ se produjo un desplazamiento del área del paño ensayado (ver Figura 10). Este problema se debió a que se produjo una falla en la unión del listón interior y el bastidor de álamo, pero cabe consignar que fue el único caso, sin embargo, este problema ayudó a plantear mejoras en el sistema de armado del panel utilizando un listón interior de 1 " $\times 1 \frac{1 / 2}{}$ ", entre las cañas en vez de uno $1 / 2$ " $\times 1 \frac{1 / 2}{}$ ". Además, se debe considerar que dada la naturaleza del armado del panel, el único paño que resultó afectado fue donde se produjo el impacto, los otros cinco cuadrantes permanecieron en las mismas condiciones iniciales, en conclusión empleando el mismo material de tierra y caña, se puede

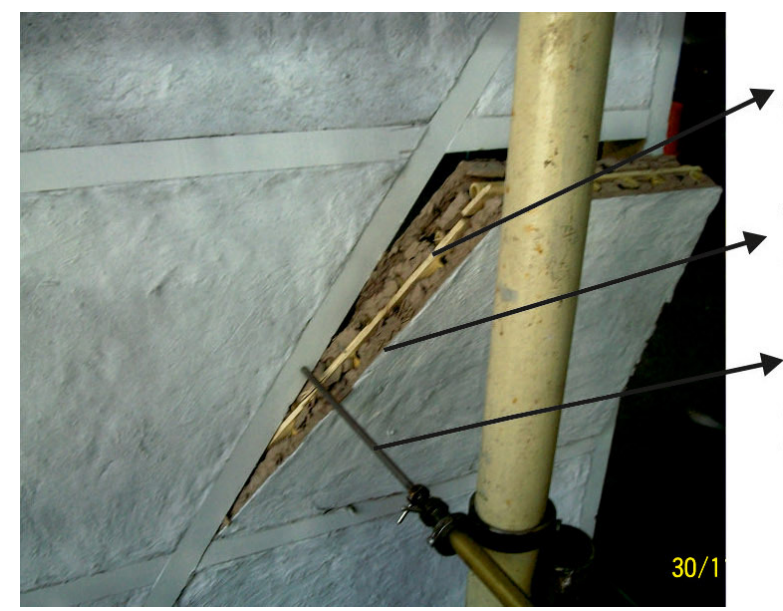

Vista del listón de 1/2" x 1 1/2" arrancado, donde se toman las cañas.

Vista del relleno de barro desplazado, pero intacto.

Vista del deflectómetro que mide la flecha, en este caso de la diagonal, que no tuvo desplazamiento (Tabla 4) 
reparar dicha área afectada sin comprometer la seguridad estructural.

\section{CONCLUSIONES}

A partir de los ensayos realizados en los suelos, se pudo concluir que la mezcla que menor retracción lineal presentó fue la correspondiente a Salto de las Rosas más un agregado del $10 \%$ del peso del suelo original en arena y el 1,5\% del peso del suelo original en peso de fibra vegetal.

Respecto al comportamiento térmico, la transmitancia térmica medida experimentalmente para una quincha de 0,094 m de espesor es de $2,64 \mathrm{~W} / \mathrm{m}^{2} \mathrm{~K}$ entre superficies. Si se adiciona una placa de poliestireno expandido de $0,005 \mathrm{~m}$, éste cambia a $2,02 \mathrm{~W} / \mathrm{m}^{2} \mathrm{~K}$. Al agregar los coeficientes de película se obtiene la transmitancia global, siendo para el panel sin aislamiento de $1,82 \mathrm{~W} / \mathrm{m}^{2} \mathrm{~K}$ y para el panel con aislamiento es $1,51 \mathrm{~W} / \mathrm{m}^{2} \mathrm{~K}$, esta última cumple con el requisito de que la transmitancia global debe ser como mínimo, menor o igual que $1,75 \mathrm{~W} / \mathrm{m}^{2} \mathrm{~K}$ para la zona de Mendoza, para la época de invierno.

En base a estos resultados, se concluye que la tecnología de quincha puede ser empleada en los muros de cerramientos de una vivienda y obtener un ambiente interior térmicamente confortable, pero se debe continuar investigando para mejorar la incorporación de aislamiento térmico.

El comportamiento mecánico muestra que los paneles de quincha cumplen con algunas de las condiciones impuestas en la normativa de ensayo de choque blando, sufriendo una deformación permanente inferior a los 3,6 mm, que es el requerido por la Norma. Respecto de la resistencia mecánica, correspondiente a una energía de choque de $180 \mathrm{~J}$ y $360 \mathrm{~J}$, presentaron un comportamiento aceptable debido a que a pesar de sufrir microfisuras visibles durante el ensayo, en la mayoría de los casos los muros no fueron atravesados por la bolsa durante el impacto y en ninguno de los casos se comprometió la seguridad estructural del mismo. A pesar de algunas microfisuras ocasionadas en el panel, el mismo no fue dañado en forma que comprometa la seguridad estructural o la de los habitantes de la vivienda, permitiendo además realizar la reparación total del paño afectado por el choque del muro para que vuelva a su estado original.

En el futuro se espera poder continuar estudiando el comportamiento estructural de los paneles de quincha frente a cargas verticales y cargas horizontales, para obtener mayor información sobre la respuesta de este tipo de construcciones principalmente en las zonas sísmicas, las cuales, cada día se emplean con mayor frecuencia al momento de buscar una solución habitacional, por parte de las familias que no cuentan con recursos financieros para acceder a una vivienda de materiales tradicionales, como son el ladrillo cocido y el cemento. Así mismo, se pretende estudiar el mejoramiento de la transmitancia térmica de la quincha, debido que sin aislamiento está a sólo $0,07 \mathrm{~W} / \mathrm{m}^{2} \mathrm{~K}$ de cumplir con la norma térmica de Mendoza, de modo que aumentando el agregado de paja o haciendo más tupida la malla de cañas, sea posible poder alcanzar ese mínimo sin tener que añadir aislamiento térmico adicional.

\section{AGRADECIMIENTOS}

Se agradece el apoyo y colaboración de los profesionales que colaboraron durante el desarrollo de esta investigación: Técnico Fernando Buenanueva, Ingeniero Sergio Acosta, Ingeniero Claudio Dagne e Ingeniero Jonathan Cueto. Asimismo a la Agencia de Promoción Científica y Tecnológica ANPCYT, que financió parcialmente este trabajo.

\section{REFERENCIAS}

(1) R. Sánchez-Hernández, M. Súarez-Barrios, J .Ma. Martín-Pozas. (200o). Caracterización de materiales antiguos de construcción (tapial y adobe) en las iglesias de Cisneros, Villada y Boada de Campos (Palencia). Materiales de Construcción, 50(257): 33-45, doi: http://dx.doi.org/10.3989/mc.2000.v50.i257.416.

(2) Octavio-Flores, M. (2003). Técnica de entramados. En Arquitecturas de Tierra en Iberoamérica (pp. 37-53). Argentina: Habiterra-Proterra.

(3) Proterra. (2003). Técnicas mixtas de construcción con tierra. PROYECTO XIV. Tecnologías de Construcción con Tierra HABYTED Subprograma XIV. Tecnología para Viviendas de Interés Social. p. 338.

(4) Gutiérrez, Aníbal. (1984). Sistema constructivo «Quincha Prefabricada». Informes de la Construcción, 36(361): 25-34, doi: http://dx.doi.org/10.3989/ic.1984.v36.i361.1962.

(5) Cuitiño, G., Esteves, A., Rotondaro, R., Maldonado, G. (2009). Análisis económico comparativo de soluciones habitacionales alternativas con quincha respecto de las construcciones tradicionales. Avances en Energías Renovables y Medio Ambiente, 13: 23-29.

(6) Blondet, M., Vargas, J., Tarque, N., Iwaqui, C. (2011). Construcción sismorresistente en tierra: la gran experiencia contemporánea de la Pontificia Universidad Católica del Perú. Informes de la Construcción, 63(523): 41-50, doi: http:// dx.doi.org/10.3989/ic.10.017.

(7) Minke, G. (2008). Manual de construcción en tierra ( $3^{\text {a }}$ edición en castellano). p. 222. Editorial Fin de siglo.

(8) Bestraten, S., Hormias, E., Altemir, A. (2011). Construcción con tierra en el siglo XXI. Informes de la Construcción, 63(523): 5-20, doi: http://dx.doi.org/10.3989/ic.10.046.

(9) Heathcote, K. (2011). The thermal performance of earth buildings. Informes de la Construcción. 63(523): 117-126, doi: http://dx.doi.org/10.3989/ic.10.024.

(10) Goodhew, S., Griffiths, R. (2005). Sustainable earth walls to meet the buildings regulations. Energy and Buildings, 37(5): 451-459, doi: http://dx.doi.org/10.1016/j.enbuild.2004.08.005.

(11) Hays, A., Matuk, S. (2003). Recomendaciones para la elaboración de normas técnicas de edificación con técnicas mixtas de construcción con tierra. Proyecto XIV.6. HABYTED Subprograma XIV - Tecnología para Viviendas de Interés Social. 
(12) Norma IRAM 11601. (2002). Aislamiento térmico de edificios. Método de cálculo - Propiedades térmicas de los componentes y elementos de construcción en régimen estacionario. Argentina: IRAM.

(13) Esteves, A., Ganem, C., Fernández, E., Mitchell, J. (2003). Thermal Insulating Material for Low-Income Housing. En 2oth Conference on passive and Low Energy Architecture, Santiago de Chile. Editado en CD: art.11.

(14) Cuitiño, G., Rotondaro, R., Esteves, A., Maldonado, G. (2010). Clasificación y estudio experimental del comportamiento de suelos mendocinos para la construcción de quinchas. Avances en Energías Renovables y Medio Ambiente. Vol. 14.

(15) Cid, J., Mazarrón, F. R., Cañas, I. (2011). Las normativas de construcción con tierra en el mundo. Informes de la Construcción, 63 (523): 159-169, doi: http://dx.doi.org/10.3989/ic.10.011.

(16) Rodríguez, M. A., Saroza, B. (2006). Identificación de la composición óptima del adobe como material de construcción de una escuela en Cuba. Materiales de Construcción, 56(282): 53-62, doi: http://dx.doi.org/10.3989/mc.2006.v56. i282.27.

(17) Naccarato, J. C. El cultivo de la caña Arundo donax en Mendoza, Argentina. http://www.gonzalezreeds.com/images/ editorial/cultivo-de-la-cana-arundo-donax-mendoza-argentina.pdf

(18) ISO. (1991). Norma ISO 8302:1991 Thermal insulation - Determination of steady state thermal resistance and related properties. Guarded hot plate apparatus. International Organization for Standardization.

(19) ASTM International. (1997). ASTM C177 Standard Test Method for Steady - State Heat Flux Measurements and Thermal Transmisión Properties by jeans of the Guarded Hot Plate Apparatus.

(20) IRAM. (1995). IRAM 11559 Determinación de la conductividad térmica y propiedades conexas en régimen estacionario. Método de la placa caliente con guarda. Buenos Aires: IRAM.

(21) ASTM International. (2003). ASTM 695 Standard Test Method of Measuring Relative Resistance of Wall, Floor, and Roof Construction to Impact Loading.

(22) IRAM. (2007). IRAM 11.596 Método de ensayo de impacto sobre probeta vertical. Buenos Aires: IRAM.

(23) Emmedue Continental S.L. (2009). Tecnología Avanzada de Construcción. Sistema constructivo MK2. Registro Mercantil de Madrid, Tomo 19.093, Libro o, Sección 8, Hoja M-333930.

(24) Bielza, J. M. (1997). Guía UEAtc para la evaluación técnica de los choques sobre obras verticales opacas. Informes de la Construcción, 49(449): 52-72, Nota Técnica.

(25) IRAM. (1991). IRAM 11.585 Paneles para muros y tabiques en edificios. Buenos Aires: IRAM.

(26) Fernández, E., Esteves, A. (2004). Conservación de energía en sistemas autoconstruidos. El caso de la Quincha mejorada. Avances en Energías Renovables y Medio Ambiente, 8(1): 121-125.

(27) IRAM. (2002). IRAM 11.601 Aislamiento térmico de edificios. Métodos de cálculo - Propiedades térmicas de los componentes y elementos de construcción en régimen estacionário. Buenos Aires: IRAM.

(28) IRAM. (1996). IRAM 11603 Acondicionamiento térmico en edificios. Clasificación bioambiental de la República Argentina. Buenos Aires: IRAM. 